\title{
Identification of Endogenous/transfected Synaptic Proteins in Primary Neuronal Culture by a High-yield Immunogold Labeling
}

\author{
Xinran Liu, Weiping Han, Thomas Biederer, Ege Kavalali and Thomas Südhof \\ Center for Basic Neuroscience, University of Texas Southwestern Medical Center, Dallas, \\ TX 75390
}

Synapses are specialized intercellular junctions between nerve cells. Neuronal signals are transmitted from the nerve terminal of the presynaptic cells to the postsynaptic target cells, this process is mediated by neurotransmitter molecules released from the synaptic vesicles. Understanding the function of the proteins that are involved in the synaptic formation and transmission requires the precise ultrastructural localization in addition to biochemical and electrophysiological approaches.

Given the fact that central synapses are so densely packed in brain, it is very difficult to achieve a uniform access for antibody to reach its target while keeping the subcellular structure distinguishable, furthermore, the quantitation of immunoreactivity is often complex and tedious. Contrarily, the primary neuronal culture whose synaptic properties are physiologically relevant to brain in situ [1] can sometimes be a good model to study the morphological characteristics of the synaptic proteins, because we are dealing with only a single-layer cells, and the procedure is generally reproducible. We have developed a simple and efficient method of a pre-embedding immunogold labeling to study the distribution of the proteins in primary hippocampal cultures. This method does not require advanced equipment and the labeling yield rate is high with relatively well-preserved morphology of synaptic structure, i.e., cell membrane and vesicles.

Here we present the immunoEM labeling data from some of our recent studies: 1) We have localized a key protein, SynCAM (synaptic adhesion molecule) to synapses, this protein plays a major role in the formation of synapses [2]; 2) We have examined the distribution of the transfected synaptotagmin I, a synaptic vesicle protein that functions as a $\mathrm{Ca}^{2+}$ sensor during neurotransmitter release [3]. By using antibodies against GFP, we were able to clearly identify the transfected synapses ultrastructurally and compare the detailed spatial expression of the normal and mutant protein in synapses.

\section{References:}

[1] T. Schikorski and C. Stevens, J Neurosci 17 (1997) 5858.

[2] T. Biederer et al., Science, 297 (2002) 1525.

[3] T. Südhof and J. Rizo, Neuron, (1996) 379. 


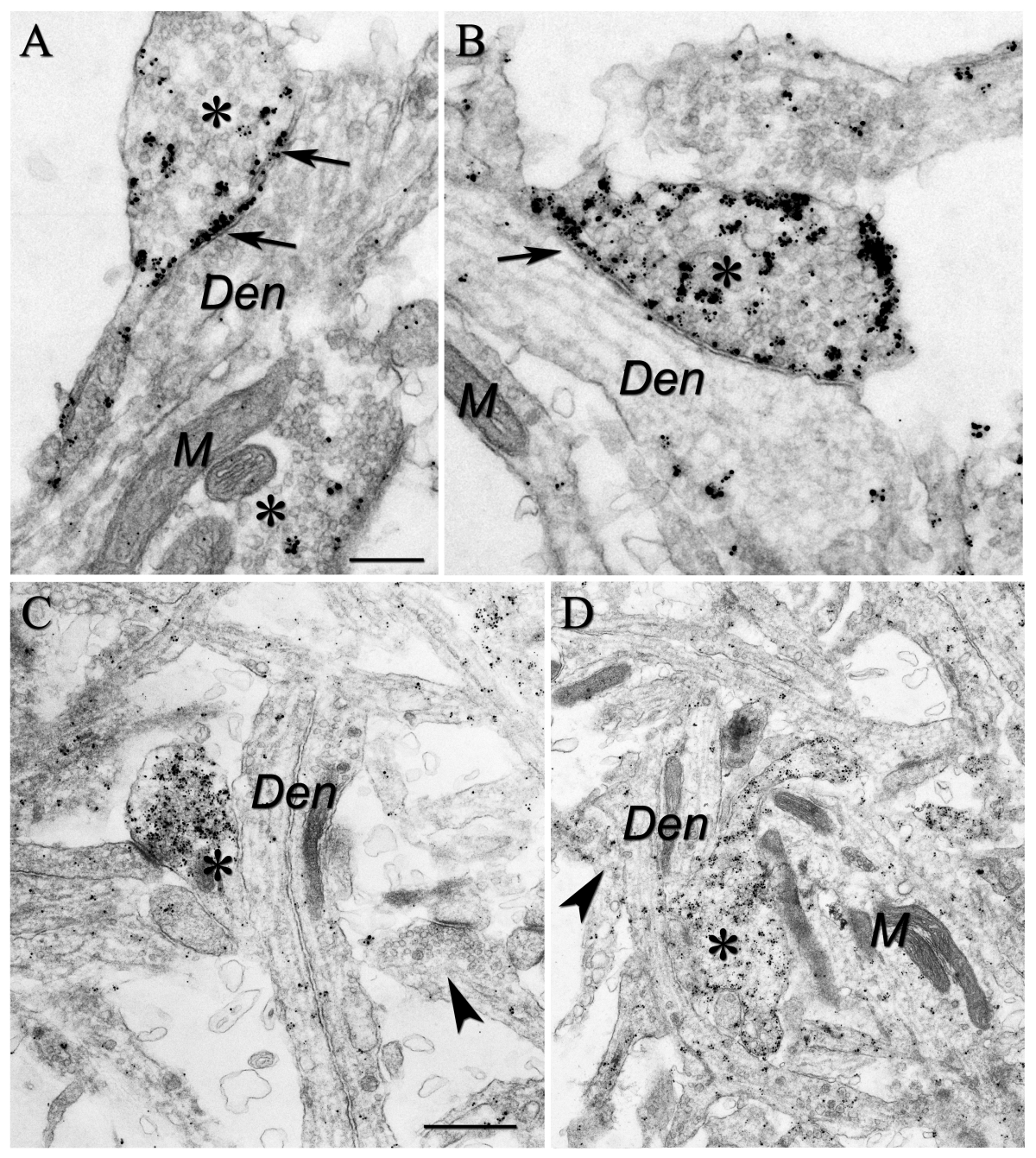

Figs A., B. Localization of SynCAM to synapses by immunogold labeling Electron micrographs of representative synaptic terminals in cultured hippocampal neurons stained with anti-SynCAM antibodies. Pre-synaptic plasma membrane especially at active zone (arrows) and a subset of vesicles were clearly labeled. Lack of uniform labeling of all terminals could be due to the presence of other SynCAM isoforms. Nerve terminals of presynaptic cells are indicated by asterisks. Den stands for post-synaptic dendrites. M stands for Mitochondria. Scale bar $=200 \mathrm{~nm}$.

Figs C., D. Transfected synaptotagmin I constructs were expressed exclusively in synapses of cultured neurons

C. Neurons transfected with normal synaptotagmin I; D. Cells transfected with the mutant protein. Note that the majority of labeling density is on synaptic vesicles, in contrast, presynaptic plasma membrane showed only minimal immunoreactivity in wildtype cells (C). This distribution pattern changed in mutant situation (D), The number of vesicles containing Synaptotagmin I appears reduced, while the signal that associates with cell membrane was signicantly increased, suggesting a possible alteration in synaptic distribution. Transfected synapses are indicated by asterisk; non-transfected synapses are labeled by arrowheads. Den stands for post-synaptic dentrites. M stands for Mitochondria. Scale bar $=500 \mathrm{~nm}$. 